\title{
Coding Question for Neonatology Today: December 2020
}

Kate Peterson Stanley, MD

Yesterday, your neonatology colleague, Dr. Smith, admitted via transfer from a referral hospital a newborn 24-week, 550-gram infant delivered by cesarean section secondary to maternal preeclampsia. The infant has respiratory failure due to RDS and is on mechanical ventilation. Dr. Smith made daily rounds earlier and has reported a global daily critical care code 99469. You are the attending physician on-call and are notified by nursing staff during the evening sign-out that the infant is hypotensive. Bedside exam shows a poorly perfused infant requiring increased oxygen support. After initiating a sepsis evaluation and antibiotic therapy, you obtain a CXR, which shows severe RDS. You recently completed a training program in point of care ultrasound and received privileges at your institution to use this tool. You perform a limited ultrasound evaluation, including five standard views (parasternal long- and short-axis, apical four-chamber, subcostal, and inferior vena cava views) of the infant's cardiac structure and function. Results show normal cardiac structure, including cardiac valves and inflow/outflow tracts. However, there is poor right ventricular cardiac function, elevated pulmonary pressures, and a moderate PDA with right to left shunting, and you decide to begin inotropic therapy. The total time for your evaluation is 45 minutes. In addition to documenting the infant's change in status in a progress note, you write a separate report describing the normal and abnormal echocardiographic findings in all five views. The echocardiographic images are recorded and stored in the hospital's Picture Archiving and Communications System (PACs).

In addition to the global daily critical care code, 99469, reported by Dr. Smith, the correct CPT codes are:

A. Evaluation and management of a critically ill patient, first 30-74 minutes: 99291

Limited/Follow up transthoracic echocardiography: 93308

B. Limited/follow up transthoracic echocardiography: 93308

C. Evaluation and management of a critically ill patient, first 30-74 minutes: 99291

Complete, transthoracic echocardiography: 93306

D. Complete, transthoracic echocardiography: 93306

"In addition to documenting the infant's change in status in a progress note, you write a separate report describing the normal and abnormal echocardiographic findings in all five views. The echocardiographic images are recorded and stored in the hospital's Picture Archiving and Communications System (PACs)."

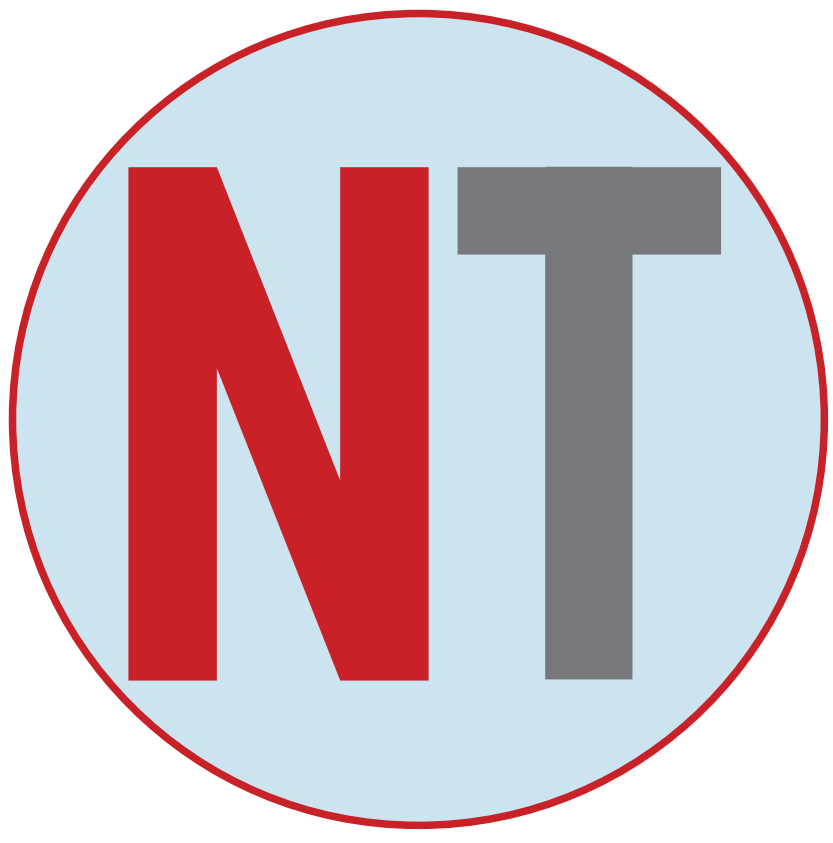

Correct Answer: B. Limited/Follow up transthoracic echocardiography: 93308

The use of point of care ultrasound (POCUS) is increasing in many NICUs to guide procedures and assist with clinical diagnosis and management. Competently trained providers may code and be reimbursed for their expertise when using this tool. Specific CPT codes vary depending on the anatomical region evaluated and whether the device is used during a specific procedure, such as thoracentesis. Three conditions are required when billing for POCUS: 1) the organ or anatomical area should be thoroughly evaluated, 2) the ultrasound findings are documented in a report within the medical record, and 3) the images are recorded and permanently archived according to local, state and federal regulations. In this specific scenario, the provider performed a limited evaluation of the infant's cardiac structure and function, documented the findings in a report, and stored the images in the hospital's PACs. Images can be retrieved as needed for clinical review, quality assurance, payer audits, or medical liability issues. Since this was a limited cardiac echocardiogram, the correct CPT code is 93308 . Limited transthoracic echocardiography is defined as a follow-up or limited study that does not provide a complete evaluation of cardiac structures. Instead, it uses two-dimensional views to answer a clinical question or evaluate a specific structure(s).

In contrast, a complete transthoracic echocardiogram includes multiple two-dimensional views that evaluate the heart's entire structure with appropriate measurements, including the left and right atria, left and right ventricles, the aortic, mitral, and tricuspid valves, the pericardium and adjacent parts of the aorta. Both codes may include M-mode imaging if performed. If a structure cannot be identified or measured, the reason for lack of visualization should be documented in the record (e.g., technical, patient instability). 
A: Incorrect: Although the limited/follow-up echocardiography CPT code is correct, the medical provider should not bill for providing additional hourly critical care. The bedside care performed and the echocardiogram are included in the global daily neonatal critical care code, 99469. In this scenario, your colleague, Dr. Smith, made daily rounds and entered the global daily care code.

C: Incorrect: The provider did not perform a complete transthoracic echocardiogram, which includes evaluating the entire structure of the heart and parts of the aorta and associated measurements. The bedside care performed and the echocardiogram are included in the global neonatal critical care admission code. In this scenario, your colleague, Dr. Smith, made daily rounds and entered the global daily care code.

D: Incorrect: The provider did not perform a complete transthoracic echocardiogram, which includes evaluating the entire structure of the heart and parts of the aorta and associated measurements.

\section{ICD-10 codes would include:}

\section{Associated with CPT code 93308:}

\section{P29.0 Neonatal cardiac failure}

Q25.0 Patent ductus arteriosus

P29.3 Persistent pulmonary hypertension of the newborn

\section{Associated with CPT code 99469:}

\section{P22.0 Respiratory Distress Syndrome}

P07.02 Extremely low birth weight newborn, 500-749 grams

P07.23 Extreme immaturity of newborn, gestational age 24 completed weeks

\section{P29.0 Neonatal cardiac failure}

\section{References:}

1. International Classification of Diseases. Wolfbane Cybernetic Ltd.. wolfbane.com/icd/index.html. Accessed July 13, 2020.

2. ICD9Data.com.http://www.icd9data.com/2015/Volume1/760-779/764-779/default.htm. Accessed July 13, 2020.

3. ICD10Data.com.https://www.icd10data.com/ICD10CM/ Codes/P00-P96/P05-P08. Accessed July 13, 2020.

Disclosure: The author has no disclosures.

NT
Corresponding Author:

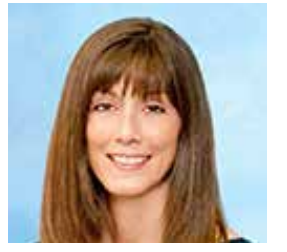

Kate Peterson Stanley, MD

Clinical Assistant Professor

Division of Neonatal-Perinatal Medicine

Department of Pediatrics

University of Michigan Medical School

Michigan Medicine

katest@med.umich.edu

\section{OPIOIDS and NAS}

When reporting on mothers, babies, and substance use

\section{LANGUAGE MATTERS}

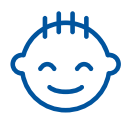

I am not an addict

was exposed to substances in utero. I am not addicted. Addiction is a set of behaviors associated with having a Substance Use Disorder (SUD).

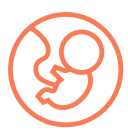

I was exposed to opioids.

While I was in the womb my mother and I shared a blood supply. I was exposed to the medications and substances she used. I may have become physiologically dependent on some of those substances.

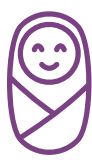

NAS is a temporary and treatable condition.

There are evidence-based pharmacological and non-pharmacological treatments for Neonatal Abstinence Syndrome.

My mother may have a SUD.

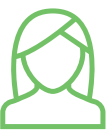

She might be receiving Medication-Assisted Treatment (MAT). My NAS may be a side effect of her appropriate medical care. It is not evidence of abuse or mistreatment.

My potential is limitless.

I am so much more than my NAS diagnosis. My drug exposure will not determine my long-term outcomes.

But how you treat me will. When you invest in my family's health and wellbeing by supporting Medicaid and Early Childhood Education you can expect that I will do as well as any of my peers!
Learn more about Neonatal Abstinence Syndrome at wWW.nationalperinatal.org 